M. Hara

Nagoya Math. J.

Vol. 75 (1979), 87-94

\title{
ON HOMEOMORPHISMS OF THE UNIT CIRCLE PRESERVING ORIENTATION
}

\author{
MASARU HARA
}

\section{Introduction}

Let $\Gamma$ denote the unit circle in the complex plane $C, C(\Gamma)$ the set of complex valued continuous functions on $\Gamma$ which is a Banach space by the sup-norm $\|\cdot\|, A(z)$ the uniform closure of all polynomials in $z$ on $\Gamma, H(\Gamma)$ the set of homeomorphisms of $\Gamma, H^{+}(\Gamma)$ the set of directionpreserving homeomorphisms and $H^{-}(\Gamma)$ the set of direction-reversing homeomorphisms. For $\psi \in H(\Gamma)$, let $A(\psi)$ denote the uniform closure of all polynomials in $\psi$ on $\Gamma$.

Any map $\psi$ belonging to $H^{-}(\Gamma)$ has the following property (A) (Browder and Wermer [2]):

(A) $\overline{A(z)+A(\psi)}=C(\Gamma)$.

The purpose of this paper is to investigate direction-preserving homeomorphisms which have the property (A). We observe the following Lemma 1 which is, in essence, contained in Browder and Wermer [2].

Lemma 1. Any map $\psi \in H^{+}(\Gamma)$ has the property (A) provided it enjoys the following property (B):

(B) $A(z) \cap A(\psi)=C$.

In view of this it is important for our purpose to classify when $\psi \in H^{+}(\Gamma)$ has the property (B). We will give one sufficient condition for $\psi \in H^{+}(\Gamma)$ to the property (B) as follows:

TheOREM 1. If, for a given map $\psi \in H^{+}(\Gamma)$, there exists a Blaschke product $B$ such that $\psi \neq B$ and the linear measure of $\{z \in \Gamma \mid \psi(z)=B(z)\}$ is positive, then $\psi$ has the property (B).

Corollary. The set of maps in $H^{+}(\Gamma)$ possessing the property (B) is

Received July 10, 1978. 
dense in the space $H^{+}(\Gamma)$.

We mention here an example of maps in $\mathrm{H}^{+}(\Gamma)$ which do not have the property (A). Consider the linear transformation

$$
U(z ; a)=\frac{z-a}{1-\bar{a} z} \quad|a|<1
$$

Clearly $U$ belongs to $H^{+}(\Gamma)$ and $A(U)=A(z)$ and a fortiori $U$ does not have the property (A). We will later give another such examples in Theorem 2, among which a typical one is following: let $B(z)=\prod_{k=1}^{n} U\left(z ; a_{k}\right)$ be with all different $a_{k}$. Then the map $(B(z))^{1 / n}$ is an example.

In connection with the welding theory of Riemann surfaces, we will give an example which has the property (B). This example will be constructed from the Jordan curve $\gamma$ in the following

TheOREM 3. On any Jordan curve $\gamma$ which contains a line segment, there dose not exist any nonconstant function which is bounded and continuous on the complex plane, analytic in the interior of and anti-analytic in the exterior of $\gamma$.

Finally we will prove the following theorem in no. 5:

THeOREM 4. If, for a given map $\psi \in H(\Gamma)$, there exists a nowhere constant function $f \in C(\Gamma)$ with the property $f(\psi)=f$, then there exists an integer $n$ with the property $\psi \circ \psi \circ \ldots \circ \psi(n$ compositions $)=z$, where a function $f \in(\Gamma)$ is said to be nowhere constant if $f$ is nonconstant on any open set of $\Gamma$.

\section{Proof of Theorem 1}

For $\alpha, \beta \in \Gamma \alpha \neq \beta$, there exist two arcs of $\Gamma$ whose end points are $\alpha$ and $\beta$. Among them we denote by $(\alpha, \beta)$ the arc starting from $\alpha$ and ending at $\beta$ in the positive direction and $[\alpha, \beta]$ the closed arc $\{\alpha\} \cup(\alpha, \beta)$ $U\{\beta\}$. Although the proof of Lemma 1 is contained in the proof of Theorem of Browder and Wermer in [2] we include it here for the sake of convenience to the readers.

Proof of Lemma 1. Assuming the conclusion is false, by the HahnBanach theorem, there exists a non-zero measure on $\Gamma$ with the following property: 


$$
\int_{|z|=1} z^{n} d \mu=0, \quad \int_{|z|=1} \psi^{n} d \mu=0 \quad(n=0,1,2, \cdots) .
$$

By the former condition of the above, we have the Riesz's representation

$$
d \mu=h(z) d z \quad h \in L^{1}(\Gamma) .
$$

If we set

$$
H(z)=\int_{[1, z]} h(\zeta) d \zeta
$$

then $H \in C(\Gamma)$ and

$$
\int_{|z|=1} z^{n} H(z) d z=\int_{|z|=1} z^{n} \int_{[1, z]} h(\zeta) d \zeta d z=\int_{|\zeta|=1} h(\zeta) \int_{[\zeta, 1]} z^{n} d z d \zeta=0 .
$$

Therefore $H \in A(z)$. Clearly $H$ is non-constant and

$$
\begin{aligned}
\int_{|z|=1} H\left(\psi^{-1}\right) z^{n} d z & =\int_{|w|=1} H(w) \psi^{n}(w) d \psi(w) \\
& =-\frac{1}{n+1} \int_{|w|=1} \psi^{n+1}(w) h(w) d w=0 .
\end{aligned}
$$

Hence $H\left(\psi^{-1}\right) \in A(z)$. If we set $H\left(\psi^{-1}\right)=G$, then $G(\psi)=H$ and $A(\psi)$. $\cap A(z) \neq C$. This is a contradiction.

The proof of Theorem 1. Suppose that $A(z) \cap A(\psi) \neq C$. There exist. non-constant functions $f \in A(z) \cap A(\psi)$ and $g \in A(z)$ with the property $f=g(\psi)$. Since $g(B) \in A(z)$ and $B(z)=\psi(z)$ for $z \in E, f(z)=g(B(z))$ for $z \in E$. By the Fatou theorem, $f=g(B)$ and $g(\psi)=g(B)$. In view of $\psi(\alpha)=B(\alpha)$ and the following Lemma $2, \psi=B$. This is a contradiction.

LEMma 2. Let the function $\tau(t)$ be monotone increasing in $\{t \geqq 0\}$ and satisfy $\tau(0)=0$. If there exists a nowhere constant function $f$ in $\{t \geqq 0\}$ with the property $f(\tau(t))=f(t)$ for $t \geqq 0$, then $\tau(t)=t$.

Proof. We set $F=\{t \geqq 0 \mid \tau(t)=t\}$. Since $F$ is closed, $\{t \geqq 0\}-F^{\prime}$ consists of countably many disjoint open intervals. Among them, we choose an arbitrary interval $(a, b)$. Assuming $\tau(t)>t$ on $(a, b)$, there exists $t_{0}$ such that $a<t_{0}<b, a<\tau\left(t_{0}\right)<b$ and $f\left(\tau\left(t_{0}\right)\right) \neq f(a)$. The sequence $\left\{\tau\left(t_{0}\right), t_{0}, \tau^{-1}\left(t_{0}\right), \tau^{-1} \circ \tau^{-1}\left(t_{0}\right), \cdots\right\}$ is contained in $(a, b)$ and monotone decreasing. We denote by $c$ the limit point of this sequence. Since $f(c)=f\left(\tau\left(t_{0}\right)\right)$, $\neq f(a)$, we conclude that $c \neq a$. On the other hand, $\tau(c)=c$, a contradiction. Similarly it does not hold that $\tau(t)<t$. 
Proof of Corollary. Given $\psi \in H^{+}(\Gamma)$ and $\varepsilon>0$, there exist two different points $\alpha$ and $\beta$ belonging to $\Gamma$ such that $\left|\psi\left(z_{1}\right)-\psi\left(z_{2}\right)\right|<\varepsilon / 2$ for $z_{1}, z_{2} \in(\alpha, \beta)$. Take a point $c$ in $(\alpha, \beta)$. We denote by $\phi$ a linear transformation which is a map from $\Gamma$ to $\Gamma$ and maps $\alpha$ to $\psi(\alpha), \beta$ to $\psi(\beta)$ and $c$ to any point of $(\psi(\alpha), \psi(\beta))$. The map $\phi$ is a finite Blaschke product. We denote by $\psi^{*}$ a map which is equal to $\phi$ on $[\alpha, \beta]$ and $\psi$ on $[\beta, \alpha]$. The map $\psi^{*}$ belongs to $H^{+}(\Gamma)$ and, by choosing $\psi^{*}(c)$ suitably, satisfies the conditions in Theorem 1 and $\left|\psi^{*}-\psi\right|<\varepsilon$.

\section{Examples which do not satisfy the property (A)}

For a finite Blaschke product $B(z)=\prod_{k=1}^{n} U\left(z ; a_{k}\right)\left|a_{k}\right|<1$, we set $\psi(z)$ $=B(z)^{1 / n}$. The map $\psi(z)$ belongs to $H^{+}(\Gamma)$ and does not satisfy the property (A) because of the following Theorem 2 . We denote by $L^{1}(\Gamma)$ the set of integrable functions on $\Gamma, C^{1}(T)$ the set of continuously differentiable functions on $\Gamma$ and $H^{1}(|z|<1)$ the subset of $L^{1}(\Gamma)$ with the following property:

$$
\int_{|z|=1} f(z) z^{n} d z=0, \quad n=0,1,2, \cdots
$$

For a function $f(z)$ defined on $\Gamma$ we denote by $\frac{\delta f}{\delta z}$ the limit

$$
\lim _{y \rightarrow z} \frac{f(y)-f(z)}{y-z}
$$

if it exists. The differential operator $\frac{\delta}{\delta z}$ has the following properties:

$1^{\circ} \quad$ For $z=e^{i \theta}, \frac{\delta f}{\delta z}(z)=-i e^{-i \theta} \frac{d}{d \theta}\left(f\left(e^{i \theta}\right)\right)$.

$2^{\circ} \quad$ For $f \quad C^{1}(\Gamma), \int_{[1, z]} \frac{\delta f}{\delta z}(z) d z=f(z)-f(1)$.

$3^{\circ}$ If $f$ is analytic at $z \in \Gamma$, then $\frac{\delta f}{\delta z}(z)=\frac{d f}{d z}(z)$.

$4^{\circ}$ For $f \in C^{1}(\Gamma)$ and $\phi \in H(\Gamma) \cap C^{1}(\Gamma)$, if we set $z=\phi(\zeta)$,

$$
\frac{\delta}{\delta \zeta}\{f(\phi(\zeta))\}=\frac{\delta f}{\delta z}(\zeta)
$$

Theorem 2. If a map $\psi \in H^{+}(\Gamma)$ is conformal on some neighborhood of $\Gamma$ and there exists a non-constant function $f(z)$ on $\Gamma$ with the following 
property:

$$
\frac{\delta f}{\delta z}(z) \in H^{1}(|z|<1) \quad \text { and } \quad f(\psi) \in A(z)
$$

then the map $\psi$ does not have the property (A).

Proof. We set

$$
d \mu=\frac{\delta}{\delta z}\{f(\psi(z))\} d z
$$

We will show that the measure $\mu$ satisfies

$$
\int_{|z|=1} z^{n} d \mu=0, \quad \int_{|z|=1} \psi^{n} d \mu=0 \quad(n=0,1,2, \cdots) .
$$

Since $f(\psi) \in A$, we have

$$
\begin{aligned}
\int_{|z|=1} z^{n} d \mu & =\int_{|z|=1} z^{n} \frac{\delta}{\delta z}\{f(\psi(z))\} d z \\
& =-n \int_{|z|=1} f(\psi(z)) z^{n-1} d z=0 .
\end{aligned}
$$

We set

$$
g(z)=\int_{[1, z]} \zeta^{n} \frac{\delta}{\delta \zeta}(\zeta) d \zeta
$$

By the former property of $f$, the function $g(\dot{z})$ belongs $A(z)$. Therefore

$$
\int_{|z|=1} z^{m} g(z) d z=0 \text { and } \frac{\delta g}{\delta z}=z^{n} \frac{\delta f}{\delta z} .
$$

Since the map $\psi$ is conformal on $\Gamma$,

$$
\frac{\delta}{\delta \zeta}\{f(\psi(\zeta))\}=\frac{\delta f}{\delta z}(\psi(\zeta)) \frac{d \psi}{d z}(z)
$$

and

$$
\begin{aligned}
\frac{\delta}{\delta \zeta} g(\psi(\zeta)) & =\frac{\delta g}{\delta z}(\psi(\zeta)) \frac{d \psi}{d z}(\zeta) \\
& =\psi^{n}(\zeta) \frac{\delta f}{\delta z}(\psi(\zeta)) \frac{d \psi}{d z}(\zeta)=\psi^{n}(\zeta) \frac{\delta}{\delta \zeta}\{f(\psi(\zeta))\}
\end{aligned}
$$

Therefore 


$$
\begin{aligned}
\int_{|\zeta|=1} \psi^{n}(\zeta) d \mu(\zeta) & =\int_{|\zeta|=1} \psi^{n}(\zeta) \frac{\delta}{\delta \zeta}\{f(\psi(\zeta))\} d \zeta \\
& =\int_{|\zeta|=1} \frac{\delta}{\delta \zeta}\{g(\psi(\zeta))\} d \zeta=0 .
\end{aligned}
$$

\section{Welding}

Given a Jordan curve $\gamma$ on the complex plane, we denote by $\Omega\left(\Omega^{*}\right.$, resp.) the interior of $\gamma$ (the exterior of $\gamma$, resp.), by $D\left(D^{*}\right.$, resp.) the interior of $\Gamma$ (the exterior of $\Gamma^{*}$, resp.) and by $\chi$ ( $\chi^{*}$, resp.) a Riemann's conformal map from $D\left(D^{*}\right.$, resp.) to $\Omega\left(\Omega^{*}\right.$, resp.) which is also a homeomorphism on the closure of the given region. From now on we assume that $\chi(1)=\chi^{*}(1)$. If we set

$$
\psi\left(e^{i \theta}\right)=\chi^{*-1} \circ \chi\left(e^{i \theta}\right),
$$

then $\psi \in H^{+}(\Gamma)$. We denote by $H_{w}^{+}(\Gamma)$ all of $\psi \in H^{*}(\Gamma)$ with $\psi=\chi^{*^{-1}} \circ \chi$ for some Jordan curve $\gamma$. By the theorem of Oikawa [5], if we define a map $\psi$ by $z^{3}$ on $\left[1, e^{2 \pi i / 5}\right]$ and $\sqrt{z}$ on $\left[e^{2 \pi t / 5}, 1\right]$ whose branches are chosen in such a way that the map $\psi$ is continuous, then $\psi \in H^{+}(\Gamma)$ and $\psi$ $\notin H_{w}^{+}(\Gamma)$. For $\psi \in H_{w}^{+}(\Gamma)$, there exist infinitely many Jordan curves corresponding to $\psi$, and among them we choose a certain $\gamma$ and $\gamma^{\prime}$. Then there is a homeomorphism $\Phi$ on $C$ which maps $\gamma$ onto $\gamma^{\prime}$ and the interior of $\gamma$ (the exterior of $\gamma$, resp.) onto the interior of $\gamma^{\prime}$ (the exterior of $\gamma^{\prime}$, resp.) and is analytic off $\gamma^{*}$ The map $\Phi$ is not necessarily conformal on C. For example, if the area of $\gamma$ is positive, the map $\Phi$ is not conformal for some $\gamma$ ([5]). By the welding theory ([5]), it is sufficient for $\psi \in H^{+}(\Gamma)$ to belong to $H_{w}^{+}(\Gamma)$ that the map $\psi$ has the following condition: for any $z \in \Gamma$, there exist $\varepsilon>0$ and $\rho>0$ (dependant on $z$ ) such that for any $\zeta$ and $t$ with $\left(\zeta e^{-i t}, \zeta e^{i t}\right) \subseteq\left(z e^{-i \varepsilon}, z e^{i \epsilon}\right)$

$$
\frac{1}{\rho} \leqq\left|\frac{\psi\left(\zeta e^{i t}\right)-\psi(\zeta)}{\psi(\zeta)-\psi\left(\zeta e^{-i t}\right)}\right| \leqq \rho .
$$

By virtue of this theorem, if $\psi$ belongs to $C^{1}(\Gamma)$ and satisfies $\frac{\delta \psi}{\delta z}(z) \neq 0$, or if $\Gamma$ is divided into finite intervals and on each interval $\psi$ is equal to a linear transformation, then $\psi$ belongs to $H_{w}^{+}(\Gamma)$.

DEFINITION. If there exist no non-constant functions that are bounded and continuous on $C$, analytic in the interior of $\gamma$ and anti-analytic in 
the exterior of $\gamma$, then we denote the fact by $\gamma \in 0$.

Lemma 3. Given $\psi \in H_{w}^{+}(\Gamma)$ and choose a Jordan curve $\gamma$ correspondent to $\psi$. Then $\psi$ has the property (B) if and only if $\gamma \in 0$.

Proof. We suppose that $f=g(\psi)$, where $f$ and $g \in A$. Since $f$ $=g\left(\chi^{*-1} \circ \chi\right)$ on $\Gamma$, we have $f\left(\chi^{-1}\right)=g\left(\chi^{*^{-1}}\right)=g\left(1 /\left(\bar{\chi}^{*}\right)^{-1}\right)$ on $\gamma$. Therefore, by observing that the function is equal to $f\left(\chi^{-1}\right)$ in the interior of $\gamma$ and $g\left(1 /\left(\bar{\chi}^{*}\right)^{-1}\right)$ in the exterior of $\gamma$, we see the validity of our lemma.

TheOREM 3. Any Jordan curve containing a line segment satisfies $\gamma \in 0$.

Proof. We suppose that the line segment is on the real axis. There exists an open disk $D$ such that the center of $D$ is on the real axis and $D$ does not have common points with $\gamma$ except for the line segment. We denote by $\Omega$ the interior of $\gamma, \Omega^{*}$ the exterior of $\gamma$ and $\Omega_{1}^{*}$ the domain obtained from $\Omega^{*}$ by reflecting it with respect to the real axis. We take a function $f$ which is bounded and continuous on $C$, analytic in the interior of $\gamma$ and anti-analytic in the exterior of $\gamma$. The function $f(\bar{z})$ is analytic in $\Omega_{1}^{*}$ and continuous on $\bar{\Omega}_{1}^{*}$. By considering $f(z)$ and $f(\bar{z})$ in $\Omega \cap D$, we see that $f(z)$ and $f(\bar{z})$ satisfy $f(z)=f(\bar{z})$ on $\partial \Omega \cap D$ and are analytic in $\Omega \cap D$. By Fatou's theorem, $f(z)=f(\bar{z})$ in $\Omega \cap D$ and $f(z)$ is analytic on $\Omega_{1} \cap \Omega^{*}$. We denote by $\Omega_{1}$ the domain obtained from $\Omega$ by reflection and by $\Omega^{\prime}$ the component of $\overline{\Omega \cup \Omega_{1}}{ }^{c}$ containing the point at infinite. The domain $\Omega^{\prime}$ is a Jordan region, whose boundary will be denoted by $\gamma^{\prime}$. The Jordan curve $\gamma^{\prime}$ is symmetric with respect to real axis. If two points $z_{1}$ and $z_{2}$ on $\gamma^{\prime}$ satisfy $z_{2}=\bar{z}_{1}$, then one of them is on $\gamma$. We may suppose that $z_{1} \in \gamma$. There exists a curve $\ell$ which connects the center of $D$ with $z_{1}$ and is contained in $\Omega^{*}$ except for its end points. If we denote by $\ell_{1}$ the curve obtained from $\ell$ by reflection with respect to the real axis, $\ell_{1}$ connects the center of $D$ with $z_{2}$ and is contained in $\Omega_{1}^{*}$ except for its end points. Since $f(z)=f(\bar{z})$ in $\Omega_{1}^{*}, f\left(z_{1}\right)=f\left(z_{2}\right)$. Hence $f$ is analytic $\Omega^{\prime}$, bounded and continuous on $\bar{\Omega}^{\prime}$ and satisfies $f(z)=f(\bar{z})$ on $\gamma^{\prime}$. Since $\Omega^{\prime}$ is symmetric, the analytic function $f(z)$ and the anti-analytic function $f(\bar{z})$ has the same boundary values, the function $f(z)$ is constant.

\section{Proof of Theorem 4}

Since $\psi \circ \psi \in H^{+}(\Gamma)$ for $\psi \in H^{-}(\Gamma)$, we will show the theorem in the case of $\psi \in H^{+}(\Gamma)$. For simplicity we denote by $\psi^{n}$ an $n$-iterated map $\psi \circ \psi \circ$ 
$\cdots \circ \psi$.

LEMma 4. Under the assumption of Theorem 4 , if $\psi \neq z$, then $f([x, \psi(x)])$ $=f(\Gamma)$.

Proof. We will show that $f([x, \psi(x)]) \subset f([\psi(x), x])$ for any $x$ and any $\psi \in H^{+}(\Gamma)$ with $f(\psi)=f$. Then from $\psi^{-1} \in H^{+}(\Gamma)$ and $f=f\left(\psi^{-1}\right)$, it follows that the reverse inclusion holds if we take $\psi(x)$ in place of $x$. We suppose that $\alpha \in f([x, \psi(x)])$ and $\alpha \notin f([\psi(x), x])$. When three points $\alpha, \beta$ and $x$ satisfy $[x, \alpha] \subset[x, \beta]$, we say that $\alpha$ is closer to $x$ than $\beta$. We denote by $t_{0}$ the closest point to $x$ among $\{t \in[x, \psi(x)] \mid f(t)=\alpha\}$. The map $\psi$ sends $\left(x, t_{0}\right)$ to $\left(\psi(x),\left(t_{0}\right)\right)$ preserving direction. Since $f\left(\psi\left(t_{0}\right)\right)=f\left(t_{0}\right)=\alpha$ and $\alpha \notin f\left([\psi(x), x], \psi\left(t_{0}\right) \notin[\psi(x), x]\right.$ and $\psi\left(t_{0}\right) \in[x, \psi(x)]$. If $t_{0}=\psi\left(t_{0}\right)$, then from Lemma 2 it follows that $\psi=z$. We may assume that $\psi\left(t_{0}\right) \in\left(t_{0}, \psi(x)\right)$. It follows that $t_{0} \in\left(\psi(x), \psi\left(t_{0}\right)\right)$ and $\psi^{-1}\left(t_{0}\right) \in\left(x, t_{0}\right)$. Since $f\left(\psi^{-1}\left(t_{0}\right)\right)=\alpha$, this contradicts that $t_{0}$ is the closest to $x$.

Proof of Theorem 4. If there exist a point $x \in \Gamma$ and an integer $n$ with $\psi^{n}(x)=x$, then $\psi^{n}=z$ because of $\psi^{n} \in H^{+}(\Gamma)$ and Lemma 2. We now discuss the rest. Given $x_{0} \in \Gamma$, we may assume that $f\left(x_{0}\right)=0$. From $f \in c(\Gamma)$, it follows that there exists a positive number $\delta$ such that $\mid f(x)$ $-f(y) \mid<1 / 2\|f\|$ for $|x-y|<\delta$. The sequence $\left\{\psi^{n}\left(x_{0}\right)\right\}$ has the following property: (1) if $m \neq n, \psi^{m}\left(x_{0}\right) \neq \psi^{n}\left(x_{0}\right)$, (2) $f\left(\psi^{n}\left(x_{0}\right)\right)=f\left(x_{0}\right)=0$. If we take $m$ and $n$ with $\left|\psi^{m}\left(x_{0}\right)-\psi^{n}\left(x_{0}\right)\right|<\delta$, then any $y \in\left(\psi^{m}\left(x_{0}\right), \psi^{n}\left(x_{0}\right)\right)$ satisfies $|f(y)|<1 / 2\|f\|$. But from $\psi^{n-m} \in H^{+}(\Gamma)$ and Lemma 4 it follows that $f\left(\left[\psi^{m}\left(x_{0}\right), \psi^{n}\left(x_{0}\right)\right]\right)=f(\Gamma)$, this is a contradiction.

\section{REFERENCES}

[1] L. Ahlfors, Lectures on quasiconformal mappings. Van Nostrand, 1966.

[2] A. Browder and J. Wermer, A method for constructing Dirichlet algebras, Proc. Amer. Math. Soc., 15 (1964), 546-552.

[ 3 ] N. Dunford and J. Schwartz, Linear operater, Part 1, Interscience, 1958.

[4] J. Garnett and A. O'Farrell, Sobolev approximation by a sum of subalgebras on the circle, Pacific J. Math., 65 (1976), 55-63.

[5] K. Oikawa, Welding of polygons and the type of Riemann surfaces, Kōdai Math. Sem., 13 (1951), 37-52.

\section{Mathematical Institute}

Division of General Education at

Faculty of Science and Engineering

Meijô University 